\title{
The gas drag in a circular binary system
}

\author{
P. Ciecieląg ${ }^{1,3}$, S. Ida ${ }^{2}$, A. Gawryszczak ${ }^{1}$, and A. Burkert ${ }^{3}$ \\ ${ }^{1}$ Copernicus Astronomical Center, Bartycka 18, 00-716 Warsaw, Poland \\ e-mail: pci@camk.edu.pl \\ 2 Department of Earth and Planetary Sciences, Tokyo Institute of Technology, Ookayama, Meguro-ku, Tokyo 152-8551, Japan \\ 3 University Observatory Munich, Scheinerstr. 1, 81679 Munich, Germany
}

Received 12 February 2007 / Accepted 27 March 2007

\begin{abstract}
We investigate the motion of massless particles orbiting the primary star in a close circular binary system with particular focus on the gas drag effects. These are the first calculations with particles ranging in size from $1 \mathrm{~m}$ to $10 \mathrm{~km}$, which account for the presence of a tidally perturbed gaseous disk. We have found numerically that the radial mass transport by the tidal waves plays a crucial role in the orbital evolution of particles. In the outer region of the gaseous disk, where its perturbation is strongest, the migration rate of a particle for all considered sizes is enhanced by a factor of 3 with respect to the axisymmetric disk in radial equilibrium. Similar enhancement is observed in the damping rate of inclinations. We present a simple analytical argument proving that the migration rate of a particle in such a disk is enhanced due to the enhanced mass flux of gas colliding with the particle. Thus the enhancement factor does not depend on the sign of the radial gas velocity, and the migration is always directed inward. Within the framework of the perturbation theory, we derive more general, approximate formulae for short-term variations of the particle semi-major axis, eccentricity, and inclination in a disk out of radial equilibrium. The basic version of the formulae applies to the axisymmetric disk, but we present how to account for departures from axial symmetry by introducing effective components of the gas velocity. Comparison with numerical results proves that our formulae are correct within several percent. We have also found in numerical simulations that the tidal waves introduce coherence in periastron longitude and eccentricity for particles on neighboring orbits. The degree of the coherence depends on the particle size and on the distance from the primary star, being most prominent for particles with $10 \mathrm{~m}$ radius. The results are important mainly in the context of planetesimal formation and, to a lesser degree, during the early planetesimal accretion stage.
\end{abstract}

Key words. binaries: close - waves - planetary systems: formation - celestial mechanics - accretion, accretion disks methods: $N$-body simulations

\section{Introduction}

Given the number of currently known extrasolar planetary systems ( 200), it is tempting to draw some conclusions about their formation from statistical correlations. One of the basic correlations is the membership in a multiple stellar system, where about $15 \%$ of the planets have been found. Assuming that the planets are equally common around single stars and in multiple systems, this is a clear discrepancy with the observed frequency of the multiplicity of solar type stars in the solar neighborhood, which is about 60\% (Duquennoy \& Mayor 1991). A natural explanation is observational selection effects, because multiple systems have been usually excluded from radial velocity searches for planets. Whether it is the only source of discrepancy will remain unknown until more systematic searches for planets in multiple systems have been carried out. Such searches are currently underway (e.g. Muterspaugh et al. 2006). It seems reasonable, however, to expect that the planet formation process is influenced by companion stars. Distant companions (say, with periastra farther than $100 \mathrm{AU}$ ) do not affect planetary systems too much, but several extrasolar planets in binary systems with relatively small separation (less than $100 \mathrm{AU}$ ) have also been discovered (Eggenberger et al. 2004, and references therein). In particular, three planets have been discovered in binary systems with separations of 20 AU (HD41004A b, $\gamma$ Cep b, G186 b). These close companions must have modified the structure of the protoplanetary disk (i.e. initial conditions for planet formation), as well as the dynamical evolution of planetary orbits.

Although the number of extrasolar planets in multiple systems is currently very small, some correlations seem to be statistically significant. Zucker \& Mazeh (2002) points out that all the most massive (more than 2 Jupiter masses), short-period (periods shorter than $40 \mathrm{~d}$ ) planets orbit stars from binary systems. In consequence, for planets in binaries there is no correlation between mass and period as is observed for planets around single stars. Eggenberger et al. (2004) shows that orbital eccentricities tend to be very low for short-period planets. All those differences indicate that the companion star is affecting the planet formation process.

To address planet formation in binary systems, studies of the orbital evolution of planetesimals have been done (e.g. Heppenheimer 1978; Whitmire et al. 1998; Marzari \& Scholl 2000; Quintana et al. 2002; Thébault et al. 2004; Thébault et al. 2006). In the classical scenario without a gaseous disk, the secular perturbations from the binary companion pump up orbital eccentricities of planetesimals and decelerate their accretion by reducing the gravitational focusing factor. Also, if the relative velocities exceed the escape velocity from their surface, collisions result in disruption rather than coagulation (e.g. Agnor \& Asphaug 2004), so that planetesimal accretion is inhibited. Marzari \& Scholl (2000) includes the effects of uniform gas drag in an eccentric binary system and finds strong periastron alignment of equal-size planetesimals. If the periastra are aligned, the 
relative velocities are kept low in spite of high eccentricities, and planetesimal accretion is not inhibited. However, Thébault et al. (2006) points out that the alignment angle depends on planetesimal size, and if the size distribution is introduced, the relative velocities between particles of different sizes are typically prohibitive for the collisional growth. An important result of these works is that even a small gas-drag force can significantly change the growth rate of planetesimals if combined with perturbations of the companion star. The weak point of these studies is the assumption that the gaseous disk is not perturbed, but remains stationary and axisymmetric. It is known (e.g. Papaloizou \& Pringle 1977; Lin \& Papaloizou 1979; Goldreich \& Tremaine 1979), however, that the companion induces tidal waves in the gaseous disk, which can evolve into strong spiral shocks. The density and velocity of the gas are then strongly perturbed, and the drag force acting on solid particles is different than in the stationary, axisymmetric case.

To date no calculations of particle motion in a disk with tidally induced spiral waves have been done. In a series of papers we will explore this subject, both analytically and numerically studying the orbital evolution and accretion of particles in disks perturbed by a companion star.

In this paper we consider the case of non-interacting particles orbiting in a circumprimary disk, with the perturbing companion star on a circular orbit. The particles range in size from $1 \mathrm{~m}$ to $10 \mathrm{~km}$, so our results apply to the planetesimal formation and early accretion phases. At first glance, the circular case may not seem interesting because the gravity of the companion does not induce secular effects on the particle orbit, like eccentricity forcing or periastra libration. However, we show that effects similar to those from an eccentric binary are also observed in the circular case if the perturbations of the gaseous disk are included. Furthermore, we show that the orbital evolution of particles in such a system is significantly different than in the unperturbed, axisymmetric disk. The circular case is a very good starting point since it allows us to understand the sole effect of spiral waves in the gaseous disk. The eccentric binary case will be discussed in next paper.

The paper is organized as follows. In Sect. 2 we present computational methods and input physics. Some important properties of the gaseous disk are discussed in Sect. 3. In Sect. 4 we investigate analytically and numerically the orbital evolution of a single particle. Section 5 is devoted to relative shapes and alignment of neighbouring orbits of particles. In Sect. 6 we summarize and discuss the results.

\section{Computational method}

The problem we investigated involves the solution of both gas and particle equations of motion. One approach would be to combine hydro and $N$-body schemes into a single numerical code, however, it was enough for our purpose to perform twostage simulations. We exploited the fact that in the circular binary system the pattern of spiral waves in the gaseous disk is quasi-stationary in the frame co-rotating with the secondary star. By quasi-stationarity we mean here that the time scale of its evolution is longer than the time scale of the evolution of $N$-body particles. In the first step we obtained such a quasi-stationary model of the gaseous disk and then we fed it to the $N$-body code. In this way we eliminated the temporal evolution of the gas. This was very desirable since our goal was to investigate generic effects of the spiral shocks on the motion of the solid bodies and not to perform realistic simulations of the particle growth in the binary system, a task we leave for future work.
The binary system we simulated consists of primary and secondary stars of equal mass, $M_{\mathrm{p}}=M_{\mathrm{s}}=1 M_{\odot}$ on a fixed circular orbit with the semi-major axis $a=23.4$ AU. The implied orbital period is close to $80 \mathrm{yr}$. The gaseous disk and particles are orbiting a primary star. We chose these parameters because, apart from the eccentricity, they are close to the $\alpha$ Centauri system investigated in Marzari \& Scholl (2000), and it will enable us to compare their results with ours, especially in the follow-up paper about the eccentric binary case. Note that the self-gravity is not included in either fluid or particle simulations, so it is possible to scale the models to any size.

\subsection{Hydrodynamical simulation}

Our model of the gaseous disk was evolved using an adaptive mesh refinement (AMR) code, FLASH (Fryxell et al. 2000). As a hydro solver we employed a direct Eulerian PPM (Colella \& Woodward 1984) scheme modified to conserve angular momentum. The PPM scheme combines high-order spatial interpolation with a Riemann solver and shock-capturing method that results in low numerical viscosity and sharp shock profiles. This makes PPM particularly useful for all applications requiring accurate transport of momentum in a supersonic flow environment.

The code solved Euler equations in 2D polar coordinates with the origin located at the primary star:

$\frac{\partial \Sigma}{\partial t}+\nabla \cdot(\Sigma \boldsymbol{V})=0$

$$
\frac{\partial(\Sigma \boldsymbol{V})}{\partial t}+\nabla \cdot(\Sigma \boldsymbol{V} \otimes \boldsymbol{V})+\nabla P=-G \Sigma\left(M_{\mathrm{p}} \frac{\boldsymbol{r}}{r^{3}}+M_{\mathrm{s}} \frac{\boldsymbol{r}-\boldsymbol{r}_{\mathrm{s}}}{\left|\boldsymbol{r}-\boldsymbol{r}_{\mathrm{s}}\right|^{3}}+M_{\mathrm{s}} \frac{\boldsymbol{r}_{\mathrm{s}}}{r_{\mathrm{s}}^{3}}\right)
$$

where $\Sigma, P$, and $\boldsymbol{V}=\left(V_{r}, V_{\phi}\right)$ denote surface density, surface pressure, and gas velocity at position $\boldsymbol{r}$. The secondary star was located at $\boldsymbol{r}_{\mathrm{s}}$ such that $r_{\mathrm{s}}=a$. The equation of state was locally isothermal (temperature was a fixed function of distance from the primary star), so there was no need to solve the energy equation, and a faster isothermal version of the Riemann solver was used.

The final model of the gaseous disk was obtained as follows: (1) the initial disk was set up as in the case of a single star, and it was truncated exponentially beyond a radius slightly larger than the expected tidal truncation radius; (2) during the first two orbital periods of the binary system the grid resolution was increased up to $2048 \times 768$ in $r$ and $\phi$ respectively (we used the AMR option here, but to avoid any artifacts at the edges of the refined blocks the whole grid was always refined); (3) the disk was evolved for an additional orbital period of the binary.

The Courant number was 0.5 . The grid extended radially from $0.4 \mathrm{AU}$ to $9 \mathrm{AU}$ - far enough to avoid any influence from boundaries on the most interesting region of the outer disk where the spiral waves are strongest. We took special care to minimize reflections at the inner and outer grid boundaries. For this purpose we tuned the standard outflow boundary conditions in order to reduce any discontinuities in radial direction. We also introduced a low-density hole in the first 5 radial cells at the inner boundary, which served as an additional buffer to damp the propagating waves. We stress that all these measures are necessary in order to recover the proper mass transport in the non-viscid disk.

\subsection{Orbital integration}

Our orbital integration code is based on Nbody4 (Aarseth et al. 1979). It implements the direct summation method for a self-gravity, 4th-order Hermite scheme, and block time step 
(Makino 1991). In this paper we consider motion of particles only under gravitational forces of the two stars and gas drag force, and mutual gravitational forces of planetesimals are neglected. Inter-particle forces and collisional accretion will be included in future papers.

In the reference frame located at the primary star, the corresponding equation of motion for particle $i$ with mass $m_{i}$ reads:

$\ddot{\boldsymbol{r}_{i}}=-G\left(M_{\mathrm{p}}+m_{i}\right) \frac{\boldsymbol{r}_{i}}{r_{i}^{3}}-G M_{\mathrm{s}} \frac{\boldsymbol{r}_{i}-\boldsymbol{r}_{\mathrm{s}}}{\left|\boldsymbol{r}_{i}-\boldsymbol{r}_{\mathrm{s}}\right|^{3}}-G M_{\mathrm{s}} \frac{\boldsymbol{r}_{\mathrm{s}}}{r_{\mathrm{s}}^{3}}+\boldsymbol{f}_{\mathrm{drag}, i}$,

where $\boldsymbol{r}_{\mathrm{s}}$ denotes the secondary star position. Components on the right hand side of Eq. (3) represent the gravity of the primary and secondary, the indirect term accounting for acceleration of the primary relative to the center of mass and gas drag force per unit mass, respectively. For the latter we adopt a simple formula:

$\boldsymbol{f}_{\text {drag }, i}=-A \rho|\boldsymbol{u}| \boldsymbol{u}, \quad A=\frac{1}{2 m_{i}} C_{\mathrm{D}} \pi s_{i}^{2}$,

where $s_{i}$ is the particle radius, $\rho$ - gas density, $C_{\mathrm{D}}-$ drag coefficient, and $\boldsymbol{u}$ is the particle velocity relative to the gas. The factor $A$ is constant in our simulations. Denoting the particle's velocity with $\boldsymbol{v}$ we have

$u_{r}=v_{r}-V_{r}, \quad u_{\phi}=v_{\phi}-V_{\phi}$.

We used values of $C_{\mathrm{D}}=1.4$ and internal density of particles $\rho_{\mathrm{p}}=2 \mathrm{~g} / \mathrm{cm}^{3}$. The Hermite scheme also requires the value of $\dot{f}_{\text {drag }}$, which is a minor correction that was accounted for using numerically calculated values of $\dot{\rho}$ and $\dot{\boldsymbol{u}}$.

We took special care to include gas-drag effects accurately yet efficiently in calculations. At the beginning of the orbital calculation, the grid data containing gas density and velocity were read in. During the simulation the data were rotated to match the current position angle of the secondary star (spiral pattern of the gas co-rotates with the companion), and the bi-linear interpolation was used to find the gas state at an arbitrary position of the particle.

The time step was variable but limited to a maximum of $1 /(2 \pi \cdot 64)$ yr. We tested the code with basic problems like conservation of the Jacobi constant, and properly recovered more complex results like runaway growth (Kokubo \& Ida 1996) and gas drag in a uniform disk (Inaba et al. 2001).

\subsection{The models}

Each model here is composed of the two components: gaseous disk and stellar system configuration. We used the following three gaseous disk configurations:

- axisymmetric disk in radial equilibrium or in a short equilibrium axisymmetric disk. Characterized by a simple powerlaw density and temperature radial profiles, the radial gas velocity is zero;

- axisymmetric disk in radial non-equilibrium or in a short non-equilibrium axisymmetric disk. It is similar to the equilibrium axisymmetric disk, but the radial gas velocity is artificially set to a non-zero value;

- non-axisymmetric disk, resulting from the evolution of an initially equilibrium axisymmetric disk in a circular binary system. It develops a spiral wave pattern and is naturally in radial non-equilibrium.

The stellar configuration can simply be either a single star or a circular binary system. Sometimes we refer to the models using abbreviations presented in Table 1 .
Table 1. Abbreviations of presented models.

\begin{tabular}{lcc}
\hline \hline & Single star & Binary system \\
\hline equilibrium axisymmetric disk & EA1 & EA2 \\
non-equilibrium axisymmetric disk & NA1 & NA2 \\
non-axisymmetric disk & W1 & W2 \\
\hline
\end{tabular}

We note here that only model W2 is physically consistent in the binary configurations, while only model EA2 has been investigated by other authors. Thus we concentrate on differences between models EA2 and W2. The other models are used only as a support in understanding the observed effects.

\section{Gaseous disk}

\subsection{Disk parameters and structure}

Our numerical method requires the gaseous disk to be in a state close to stationarity. It should also be minimally biased by numerical effects and should adequately recover all deviations from the Keplerian flow. To that end we used a fairly simple isothermal model, which nonetheless is close to the minimum mass solar nebula (Hayashi 1981).

The equation of state,

$P=\Sigma c_{\mathrm{s}}^{2}$

is locally isothermal, with the local sound speed $c_{\mathrm{s}}$, given by the vertical hydrostatic equilibrium condition

$c_{\mathrm{s}}=\frac{h}{r} v_{\mathrm{k}}$

where $v_{\mathrm{k}}=\sqrt{G M_{\mathrm{p}} / r}$ is the Keplerian velocity and $h$ the local half-thickness of the disk. Blondin (2000) has shown that, in sufficiently cold disks, the spiral waves at the outer edge of the disk may become unsteady. We found experimentally that for the following height profile

$h_{r} \equiv \frac{h}{r}=0.05 \cdot\left(\frac{r}{1 \mathrm{AU}}\right)^{0.5}$

the spiral pattern stays stable everywhere. Note that, although our equation of state is locally isothermal, such $h_{r}$ results in global isothermality with $c_{\mathrm{s}}=0.05 \sqrt{G M_{\mathrm{p}} /(1 \mathrm{AU})}$. The corresponding Mach number $\left(v_{\mathrm{k}} / c_{\mathrm{s}}\right)$ is 31.4 at the inner disk edge ( $r=0.9 \mathrm{AU})$, which falls to 7.6 at the outer edge ( $r=9 \mathrm{AU})$.

The initial profile of the surface density was given by the power law

$\Sigma_{i}=\Sigma_{0}\left(\frac{r}{1 \mathrm{AU}}\right)^{-1.5}$,

which is close to the MMSN model. Since self-gravity is not included in hydro simulation, the normalization $\Sigma_{0}$ can be arbitrary. To calculate the drag force (Eq. (4)) during orbital integration, the evolved surface density $\Sigma(\boldsymbol{r})$ was converted to the volume density and normalized as follows:

$\rho=2 \times 10^{-9} \frac{\Sigma / \Sigma_{0}}{h(r) / 1 \mathrm{AU}}=2 \times 10^{-9} \frac{\Sigma}{\Sigma_{0}}\left(\frac{r}{1 \mathrm{AU}}\right)^{-1.5}\left[\mathrm{~g} / \mathrm{cm}^{3}\right]$.

The initial angular velocity was corrected for the pressure gradient

$V_{\phi}=v_{\mathrm{k}}+\frac{1}{2} \frac{\partial \ln P}{\partial \ln r} \frac{c_{\mathrm{s}}^{2}}{v_{\mathrm{k}}}$

to assure the radial equilibrium $\left(V_{r}=0\right)$. 
The initial conditions described above represent our equilibrium axisymmetric disk configuration. This configuration was evolved numerically for three binary orbital periods, which is enough to develop a quasi-stable spiral pattern. This evolved disk will be referred to as the non-axisymmetric disk.

Here we have to point out that the 2D approximation introduces a certain inconsistency into both models of the gaseous disk. The problem with $2 \mathrm{D}$ hydrodynamical simulations is that the velocity field cannot be directly linked to the 3D velocity. The 2D velocity given by Eq. (11) assures radial equilibrium for a given gradient of the vertically averaged pressure. Note, however, that it is neither the vertically averaged velocity nor velocity in the equatorial plane. Proper, equatorial plane velocity is given by

$V_{\phi}=v_{\mathrm{k}}+\frac{1}{2} \frac{\partial \ln p}{\partial \ln r} \frac{c_{\mathrm{s}}^{2}}{v_{\mathrm{k}}}$

where $p=\rho c_{\mathrm{s}}^{2}$ denotes pressure in the equatorial plane. Both formulae give different results since, in general, the gradient of $p$ is different from the gradient of $P$. Unfortunately, the simulated $2 \mathrm{D}$ velocity cannot be transformed to the equatorial plane velocity. Thus an inconsistency arises: we use 2D velocities, while the density is converted to 3D one (Eq. (10)). We decided that it is better to use Eq. (11) for the axisymmetric disk model although Eq. (12) should be used: our results may not be accurate quantitatively, but at least we can compare both models qualitatively. Furthermore we expect relative results from both models to be less affected than the absolute results.

For the later considerations it is useful to define two dimensionless velocities which describe deviations from the Keplerian flow:

$\eta=\left(v_{\mathrm{k}}-V_{\phi}\right) / v_{\mathrm{k}}$,

$\kappa=-V_{r} / v_{\mathrm{k}}$.

In other words, these are velocity components of a large particle moving on a circular, Keplerian orbit relative to the gas.

To reveal the influence of spiral waves on the particle motion, we have to compare results obtained in the axisymmetric disk with those obtained in the perturbed disk at a radius where angle-averaged values of $\rho, V_{r}$, and $V_{\phi}$ are comparable. The upper panel of Fig. 1 shows the radial profiles of $\eta$ averaged over the full angle for three disk models: non-axisymmetric, axisymmetric employing formula (11), and axisymmetric employing formula (12). As we see, the profiles of the two first models are comparable up to a distance of 3 AU from the primary star. Outside of this region the deviations from the Keplerian flow grow substantially. Since the spiral waves are strongest in the outer region of the disk, in the next section we will trace the motion of the particle placed initially at 3 AU. The dash-dotted curve illustrates why the "3D velocity" is not suitable for our comparison. The density in the non-axisymmetric model at $3 \mathrm{AU}$ has grown during the simulation time by roughly $15 \%$ with respect to the initial model (see lower panel of Fig. 1). Since the migration speed due to gas drag scales linearly with the density, this difference can be easily accounted for in comparisons with the axisymmetric disk.

\subsection{Radial transport of gas}

When the spiral density waves are excited in the disk, it is no longer in radial equilibrium. Because the spiral pattern is rotating slower than the local Keplerian velocity, the dissipation at

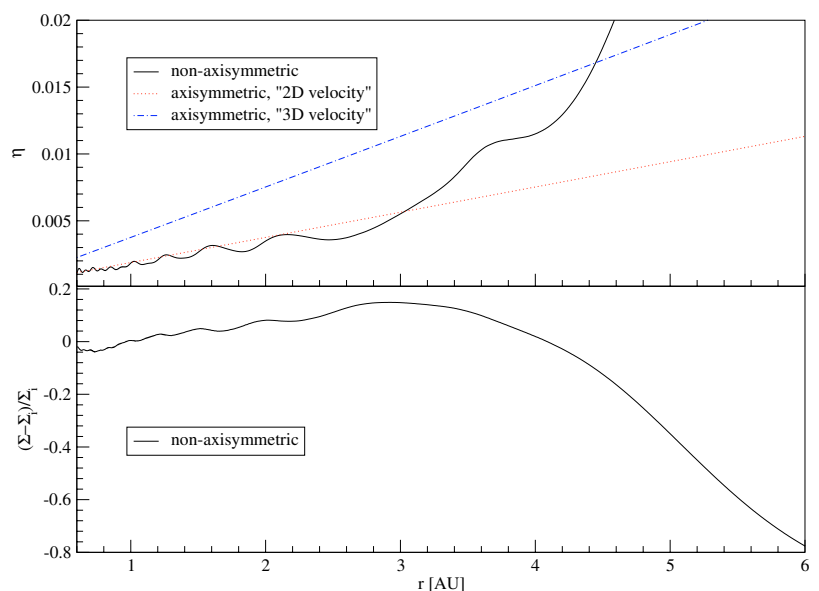

Fig. 1. Top panel: radial profiles of angle-averaged $\eta$ in nonaxisymmetric disk (solid), axisymmetric disk with "2D velocity" (Eq. (11), dotted), and axisymmetric with "3D velocity" (Eq. (12), dotdashed). Bottom panel: radial profile of angle-averaged surface overdensity, $\left(\Sigma-\Sigma_{i}\right) / \Sigma_{i}$, in a non-axisymmetric disk.

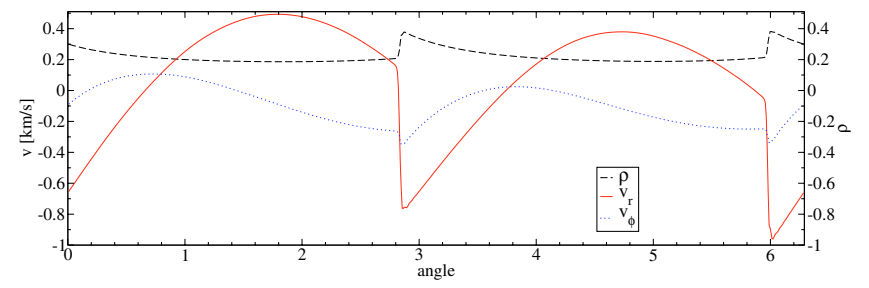

Fig. 2. Angular cross-sections of velocity components and density at $r=3 \mathrm{AU}$.

the shock leads to a decrease in angular momentum of the orbiting gas and its radial mass transport. Figure 2 shows the angular cross sections of velocity components and density at $3 \mathrm{AU}$ in the non-axisymmetric disk. Indeed, the angle-integrated mass flux calculated from those profiles is negative (inward). It can be interpreted as the result of an effective viscosity that we define as the turbulent viscosity necessary to cause the same radial mass flux. In the standard $\alpha$-disk theory, the kinematic viscosity $v$ is expressed in terms of a dimensionless parameter $\alpha$ as $v=2 / 3 \alpha c_{\mathrm{s}} h$. Assuming a steady accretion disk, i.e. $\dot{m}=-3 \pi v \Sigma$ independent of $r$, we can parametrize the mass accretion rate with the effective value of $\alpha_{\text {eff }}$ :

$\dot{m}=2 \pi r V_{r} \Sigma=-2 \pi \alpha_{\mathrm{eff}} c_{\mathrm{s}} h \Sigma$.

For the non-axisymmetric disk, the above equations must be averaged over the azimuthal angle, and finally the effective $\alpha$-viscosity is defined as

$\alpha_{\mathrm{eff}}=-\frac{2 v_{\mathrm{k}} V_{\mathrm{r}, \mathrm{eff}}}{3 c_{\mathrm{s}}^{2}}$

where

$V_{\mathrm{r}, \mathrm{eff}}=\frac{\int_{0}^{2 \pi} \Sigma V_{\mathrm{r}} \mathrm{d} \phi}{\int_{0}^{2 \pi} \Sigma \mathrm{d} \phi}$

It has been shown analytically (Spruit 1987) and numerically (Blondin 2000; Różyczka \& Spruit 1993) that the mass transport by tidal waves can be very effective. For reasonable disk parameters in close binary systems, the effective $\alpha$-viscosity in the outer parts of the disk can easily reach 0.1 or even more. It is hard to produce such high values by ordinary turbulent viscosity. 


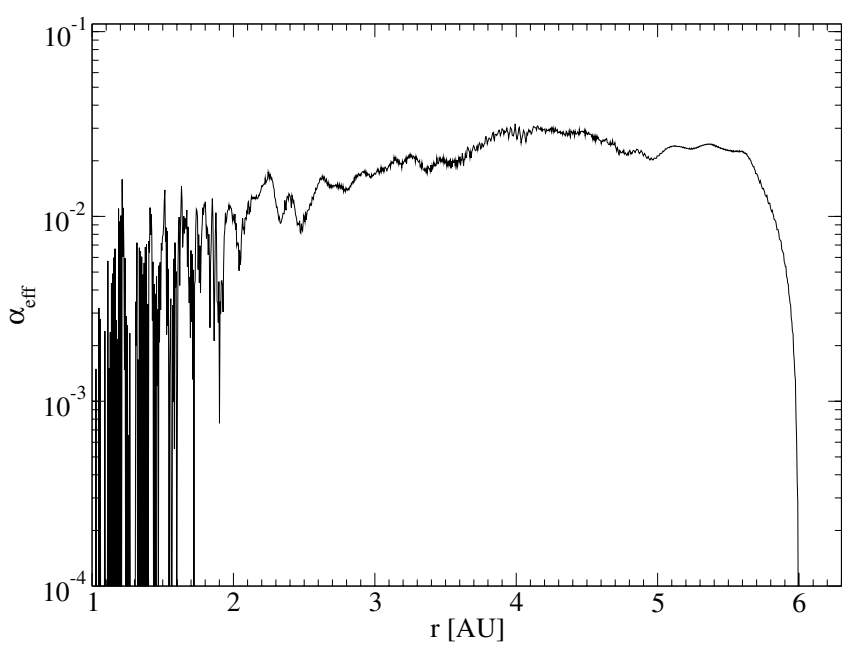

Fig. 3. Radial profile of the effective $\alpha$-viscosity.

The radial profile of $\alpha_{\text {eff }}$ in our non-axisymmetric disk is shown in Fig. 3. Close to the inner edge of the disk, it oscillates strongly because the spiral waves are very tightly wound, and the radial grid resolution is insufficient for resolving them. However in the region close to $3 \mathrm{AU}$, the resolution is sufficient, and the effective $\alpha$ may be easily found.

\section{Evolution of orbital elements}

The rate of change of orbital elements of a particle experiencing gas drag was calculated by Adachi et al. (1976, hereafter A76). Their results cannot be applied, however, to a disk in the binary system because the authors assumed that the disk is axisymmetric and stays in radial equilibrium $\left(V_{r}=0\right)$. In the binary system the disk is neither axisymmetric nor in radial equilibrium, so the evolution of orbital elements may be very different. In this section we investigate the evolution of orbital elements, first in the axisymmetric disk out of radial equilibrium, and then in the nonaxisymmetric, perturbed disk. In both cases we derive perturbative formulae which extend the ones by A76, and test them with numerical simulations.

\subsection{Non-equilibrium axisymmetric disk: analytical calculations}

First, we consider the simplest case of the particle on a nearly circular, non-inclined orbit. Let $u=\sqrt{u_{r}^{2}+u_{\phi}^{2}}$ be the value of the total relative velocity between particle and gas, where $\left(u_{r}, u_{\phi}\right)$ are radial and angular components, respectively. The particle loses specific angular momentum only due to angular component of the drag force, and for small drag (when the orbit stays nearly circular) its loss rate can be approximated as

$\frac{\mathrm{d}\left(v_{\mathrm{k}} a\right)}{\mathrm{d} t}=\frac{1}{2} v_{\mathrm{k}} \frac{\mathrm{d} a}{\mathrm{~d} t} \approx-\frac{u_{\phi} \cdot a}{\tau}$

where $v_{\mathrm{k}}$ is the Keplerian velocity at radius $a$, and $\tau$ is the stopping timescale:

$\tau=\frac{u_{\phi}}{A \rho u_{\phi} u}$.

Thus the orbit decay rate is given by the approximate formula

$\frac{\mathrm{d} a}{\mathrm{~d} t} \approx-2 A \rho a \frac{u_{\phi}}{v_{\mathrm{k}}} u$.

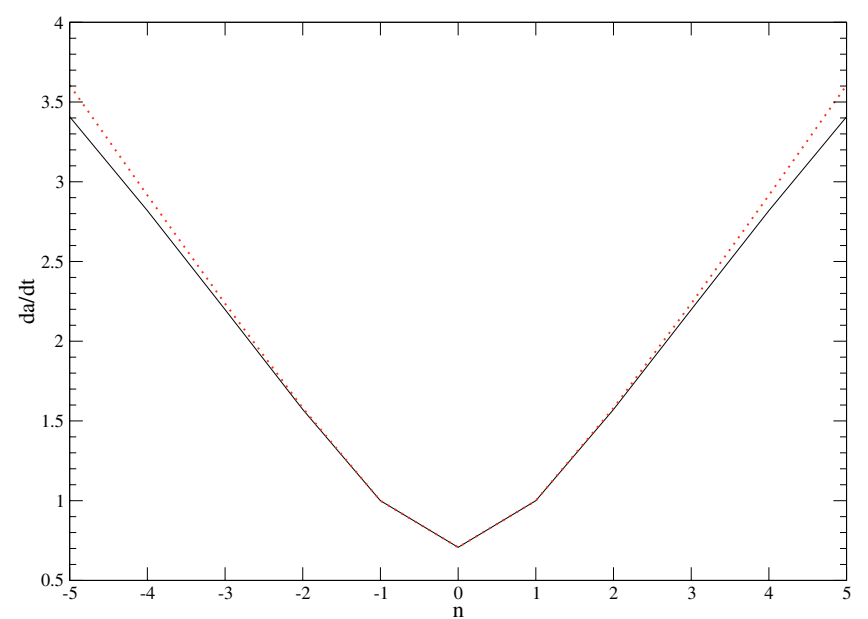

Fig. 4. Dependence of the particle migration rate (in units of $\dot{a}(n=1)$ ) on the radial gas velocity. Solid line - simulation; dotted line - analytical approximation (Eq. (23)).

There are two important factors here: $u_{\phi}$ and $\rho u$. The particle loses angular momentum when colliding with the gas at relative velocity $u_{\phi}$, but the mass flux of this gas is $\rho u$, which is how the radial gas velocity enhances the migration rate. For large enough particles moving with Keplerian velocity, we can further write

$\frac{\mathrm{d} a}{\mathrm{~d} t} \approx-2 A \rho a \eta u$.

In Appendix A we calculate the evolution of orbital elements for the general case of eccentric and inclined orbits within the framework of perturbation theory. In the limit of a circular, noninclined orbit, the general formula for orbitally averaged $\mathrm{d} a / \mathrm{d} t$ (Eq. (A.8)) reduces exactly to Eq. (21).

We would like to turn the reader's attention to the dependence on the value of total relative velocity in formula (20) (for details, see Appendix). In particular, in the limit of high radial velocity, $u_{r} \gg u_{\phi}$, we have a very surprising relation:

$\frac{\mathrm{d} a}{\mathrm{~d} t} \propto-\left|u_{r}\right|$

The migration rate is proportional to the absolute value of gas radial velocity; i.e., the particle migrates inward regardless of the direction of the radial gas flow! In order to test this result numerically we measured particle migration rates in the axisymmetric disk, while artificially varying the radial gas velocity. The particle was initially on a circular orbit with components of relative velocity $u_{\phi}$ and $u_{r}=V_{r}=n \cdot u_{\phi}$, where $n$ was an integer number between -5 and 5 . Figure 4 shows measured and predicted migration rates (normalized to $\dot{a}(n=1)=1$ ) as a function of $n$. The predicted curve is given by the formula

$\dot{a}(n)=\sqrt{\frac{1+n^{2}}{2}}$.

Indeed, the migration rates measured in the simulation for $n$ and $-n$ are the same within $1 \%$. Also the agreement with prediction (Eq. (23)) is very good, especially for small $n$. For higher values of $|n|$, formula (23) deviates slightly from results of the simulation because the orbit differs more and more from the assumed circular shape. In fact, changes in the semi-major axis and eccentricity are coupled so must be considered together. Even though the radial gas velocity does not change the angular momentum of the particle directly, it does change its eccentricity, 


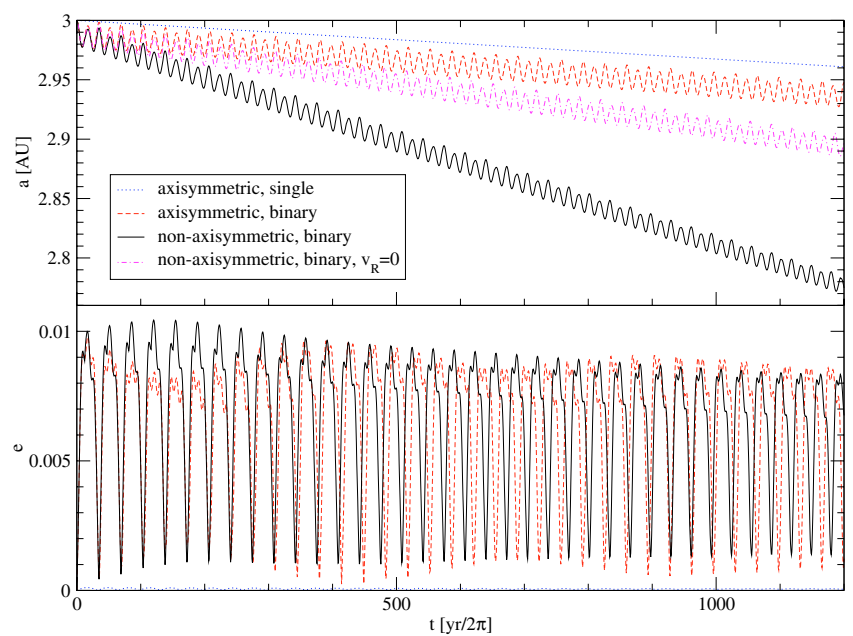

Fig. 5. Semimajor axis (upper panel) and eccentricity (lower panel) versus time for a $10 \mathrm{~m}$ particle in models: W2 (solid), EA2 (dashed), EA1 (dotted) and W2 with radial gas velocity set to zero (dash-dotted).

which in turn affects the decay rate of the semimajor axis (see Eqs. (25), (26)).

The above result concerns an idealized axisymmetric disk and aims to enhance basic effects of radial gas flow (regardless of its source), as well as to provide a proof of code validity.

\subsection{Non-axisymmetric disk: numerical and analytical results}

Now, lets have a look at the particle moving in the nonaxisymmetric disk perturbed by a companion star, and compare its motion to that in the unperturbed disk.

As a first example, we consider particle of radius $10 \mathrm{~m}$ on an initially non-inclined, circular orbit of radius $3 \mathrm{AU}$. We checked empirically that in all our models the particle of this size migrates by less than $0.2 \mathrm{AU}$ within the simulation time, so that the parameters of the gaseous disk can be regarded as roughly constant. This enables us to compare the migration speed between different disk models. The upper panel of Fig. 5 shows the evolution of the particle's semimajor axis in models W2, EA2, and EA1. As we see, the difference in migration speed of a particle in the non-axisymmetric disk is substantially enhanced with respect to the other two cases, roughly by a factor of three. The lower panel of Fig. 5 shows the corresponding evolution of eccentricity. Oscillations with the synodic period of the companion star are clearly seen. We checked that the oscillations are not damped by gas drag for particles larger than $10 \mathrm{~m}$. The amplitude of oscillations depends on the semimajor axis, and the mean eccentricity is roughly the same in both models with a companion star (only a very small decrease is observed with decreasing $a$ ). For this reason the eccentricity cannot be responsible for the difference in the migration speed. We have already shown analytically that this relatively fast migration can be induced by the radial gas velocity. In order to test this prediction numerically, we performed an orbital calculation with the non-axisymmetric disk in which the radial gas velocity was set artificially to zero. The corresponding curve in Fig. 5 clearly proves that the radial gas flow is the main factor responsible for the accelerated particle migration. Another two factors are responsible for the remaining part of the difference from the axisymmetric model. First, in the non-axisymmetric case the effective value of $\eta^{2}$ is higher due to weighting by the density (see discussion in the next paragraph). Second, due to the dissipation in spiral waves, the mean density in the simulation has increased by $15 \%$ with respect to the axisymmetric model (see Fig. 1).

How well do the above numerical results agree with the analytical approximations? The comparison is straightforward for the equilibrium axisymmetric disk. In that case formula A.8 reduces to the original formula 4.21 from A76. We have $a / \tau_{0}=$ $0.51[2 \pi \mathrm{AU} / \mathrm{yr}]$ in our disk model for the $10 \mathrm{~m}$ particle on the orbit with $a=3$ AU. Furthermore, $\eta=0.0056$, and the measured mean eccentricity is $e=0.006$. For those parameters the analytically predicted migration velocity is within $1 \%$ of the value measured in the simulation: $4.3 \times 10^{-5}[2 \pi \mathrm{AU} / \mathrm{yr}]$. This proves the accuracy of our orbital integration.

Application of formula A.8 to the non-axisymmetric disk is not as straightforward. We have found that simply inserting angular averages of $\eta$ and $\kappa$ leads to quite a large discrepancy with the value measured in simulation. This is because the density in our non-axisymmetric disk is correlated with the velocity so the approximation A.6 is not justified. Here we derive a new version of the formulae A.8-A.9 that takes such a correlation into account. To that end we modify approximation A.6 by also detaching $\rho$ from $F$ and averaging its product with $u$ separately:

$\langle\rho u F\rangle=\left\{\left\langle(\rho u)^{2}\right\rangle\right\}^{1 / 2}\langle F\rangle$,

where the orbital average, \langle\rangle is defined by Eq. (A.5). This leads to the following formulae:

$\frac{\tau_{0}^{\prime}}{a}\left\langle\frac{\mathrm{d} a}{\mathrm{~d} t}\right\rangle=-2\left[\left(\frac{5}{8}-\kappa_{\mathrm{eff}}^{2}\right) e^{2}+\frac{1}{2} i^{2}+\eta_{\mathrm{eff}}^{2}+\kappa_{\mathrm{eff}}^{2}\right]^{1 / 2} \eta$

$\frac{\tau_{0}^{\prime}}{e}\left\langle\frac{\mathrm{d} e}{\mathrm{~d} t}\right\rangle=2 \frac{\tau_{0}^{\prime}}{i}\left\langle\frac{\mathrm{d} i}{\mathrm{~d} t}\right\rangle=\left[\left(\frac{5}{8}-\kappa_{\mathrm{eff}}^{2}\right) e^{2}+\frac{1}{2} i^{2}+\eta_{\mathrm{eff}}^{2}+\kappa_{\mathrm{eff}}^{2}\right]^{1 / 2}$

which are very similar to Eqs. (A.8)-(A.9); but variables $\eta$ and $\kappa$, which enter the formula for the total relative velocity (Eq. (A.4)), have been changed to the effective values

$\eta^{2} \rightarrow \eta_{\mathrm{eff}}^{2}=\frac{\overline{(\rho \eta)^{2}}}{\bar{\rho}^{2}}$

$\kappa^{2} \rightarrow \kappa_{\mathrm{eff}}^{2}=\frac{\overline{(\rho \kappa)^{2}}}{\bar{\rho}^{2}}$.

The bar denotes here averaging over the full angle at radius $r=a$ in contrast to the orbital average defined by Eq. (A.5). Also the characteristic time scale now depends on the averaged density:

$\tau_{0}^{\prime}=\frac{1}{A \bar{\rho} v_{\mathrm{k}}(a)}$.

We note that $e$ and $i$ are not translated to the effective values because we have assumed that they remain constant during one orbital period. This might not be fulfilled for small particles that are more strongly coupled to the gas. Fortunately, the effect becomes noticeable only at the lower limit of the size range for which the drag law we used is applicable. In analogy to Eq. (28), we can define the effective $\alpha$-viscosity, and its relation to $\kappa_{\text {eff }}$ is

$\kappa_{\mathrm{eff}}=\frac{3}{2} h_{r}^{2} \sqrt{\overline{\alpha_{\mathrm{eff}}^{2}}}$.

Now we are in a position to test this result with numerical simulations. From the model W2 we measured mean $e=0.006, \eta=$ 0.0056 , and effective $\kappa_{\text {eff }}=0.027, \eta_{\text {eff }}=0.01$. For these parameters, the predicted migration velocity is $1.9 \times 10^{-4}[2 \pi \mathrm{AU} / \mathrm{yr}]$ in 


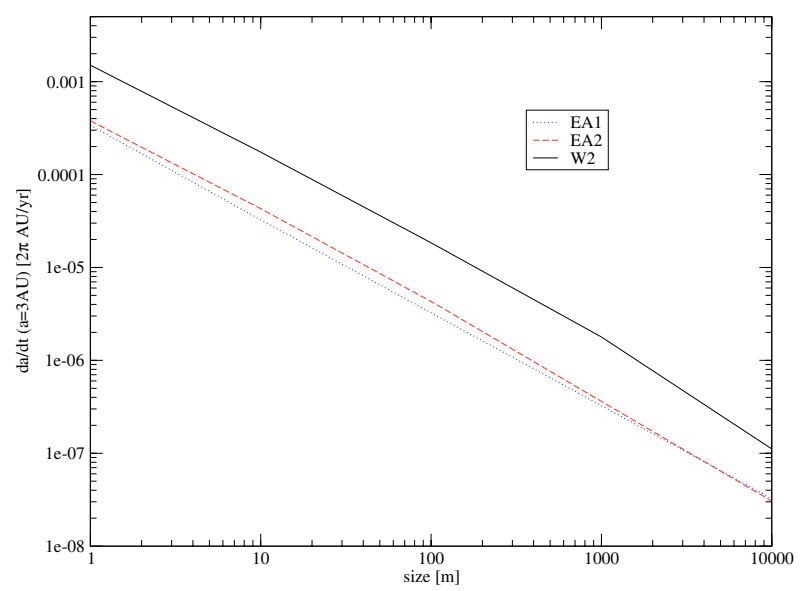

Fig. 6. Migration rate as a function of the particle size for models: W2 solid line, EA2 - dashed line, and EA1 - dotted line.

comparison to $1.8 \times 10^{-4}$ [ $2 \pi \mathrm{AU} / \mathrm{yr}$ ] measured from the simulation. Taking the number of approximations that had to be done in the analytical derivation into account, this agreement is indeed excellent.

In Fig. 6 we display the migration rates measured in simulations as a function of the particle size. As we see, the presence of the companion star does not change the migration rate in axisymmetric disks, because the excited eccentricity oscillations are small in comparison to the $\eta$ parameter that plays the major role. In the non-axisymmetric disk parameter $\kappa_{\text {eff }}$ clearly dominates other factors $\left(e, i, \eta_{\mathrm{eff}}\right)$, and thus for all considered sizes migration speed is enhanced by roughly the same factor of 3 . Of course this factor will change as $\kappa_{\mathrm{eff}}$ is changing. The value of $\kappa_{\text {eff }}$ depends on the position in the disk, disk properties, and binary separation. In particular, for a given disk model, it decreases with decreasing $r$, because the waves are weaker in the inner disk. For a discussion of the dependence of $\alpha_{\text {eff }}$ on the disk density profile and temperature (for isothermal models), please refer to Blondin (2000).

To test the formula for inclination damping (Eq. (26)), we placed a particle on an initially circular orbit at $3 \mathrm{AU}$ with inclination 0.01 . The evolution of its orbital elements in models $\mathrm{W} 1$, W2, and EA2 is shown in Fig. 7. Clearly, the inclination decreases faster in the non-axisymmetric disk. The initial difference in damping rate, measured over interval $\delta t=50 \mathrm{yr} / 2 \pi$, is about three times higher than in model EA2. In order to make a comparison with the analytical approximation (which does not account for the presence of a secondary), we ran one more model with the non-axisymmetric disk but with the gravity of the secondary star switched off (W1; see Fig. 5). The difference between models W1 and W2 is very small, which is expected since the companion star influences the inclination rather weakly. We measured the initial slope in model W1 to be $2.2 \times 10^{-5}[2 \pi / \mathrm{yr}]$, while the approximate Eq. (26) gives $2.5 \times 10^{-5}[2 \pi / \mathrm{yr}]$, which makes a $12 \%$ difference. Althrough worse than for the semimajor axis, this accuracy is still very good for an approximate formula.

The eccentricity of the particle in a binary system is set by the balance between forcing from the secondary and drag damping. As it was already shown in Fig. 5, the eccentricity of a particle on an initially circular orbit is immediately forced to oscillate with a roughly constant amplitude (0.01 at $3 \mathrm{AU})$. Comparison of the analytical formula for damping of $e$ (Eq. (26)) with simulation results in model $\mathrm{W} 2$ would require setting up the particle

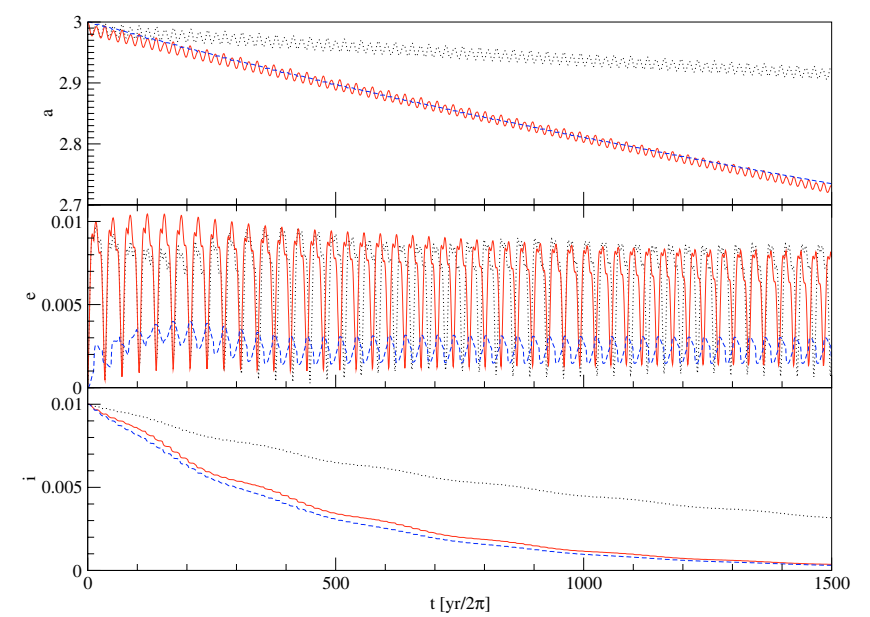

Fig. 7. Evolution of orbital elements for a particle on an initially circular, inclined orbit in models: W2 (solid line), EA2 (dotted line), and W1 (dashed line).

with initial eccentricity substantially higher than 0.01 . Since it is rather unlikely for a small particle to have such eccentricities, and in addition analytical approximation may not work well in this regime, we will not present it. We note only that the comparison of eccentricity between models W1 and W2 in Fig. 7 reveals that the spiral waves are only responsible for the lower limit of eccentricity in model W2, while the remaining contribution comes from the perturbation by the secondary.

\section{Coherence of periastra and eccentricities}

Relative shape and alignment of neighbouring orbits is a very important factor because it controls the relative velocities of the particles and thus their growth rate. Even if the companion star excites high eccentricities, it does not necessarily mean that the relative velocities are high. Marzari \& Scholl (2000) have shown that secular perturbations from an eccentric companion, combined with the gas drag, lead to the strong alignment of periastra between particles of the same size on neighbouring orbits. Since periastra are also coupled to eccentricities, the relative velocities are low. This effect of "orbital phasing" in an eccentric binary is prominent even for bodies of $100 \mathrm{~km}$ in diameter, which are commonly regarded as decoupled from the gas. It should be stressed that the collision velocities are low only between particles of the same size. Thébault et al. (2006) has shown that for distribution of sizes, even small misalignment in the periastra of particles of different sizes results in relative velocities that are high enough to prohibit collisional growth (note however that they used an equilibrium axisymmetric-disk approximation).

In this section we investigate the effects of the orbital coherence, but in the circular binary system and for smaller sizes of particles. The secular effects of eccentricity forcing and periastra alignment are not present in the circular system. However, the inclusion of a non-axisymmetric gaseous wave pattern provides an additional factor that localy perturbs orbit of the particle. Since the wave pattern co-rotates with the companion star, we may expect similar effects to the secular gravitational perturbations. To check what really happens in such systems, we performed 4 runs with non-interacting particles of sizes $1 \mathrm{~m}, 10 \mathrm{~m}, 100 \mathrm{~m}$, and $1 \mathrm{~km}$. Each run was initiated with 30000 particles on circular, non-inclined orbits distributed randomly between $0.8 \mathrm{AU}$ and $6 \mathrm{AU}$. 


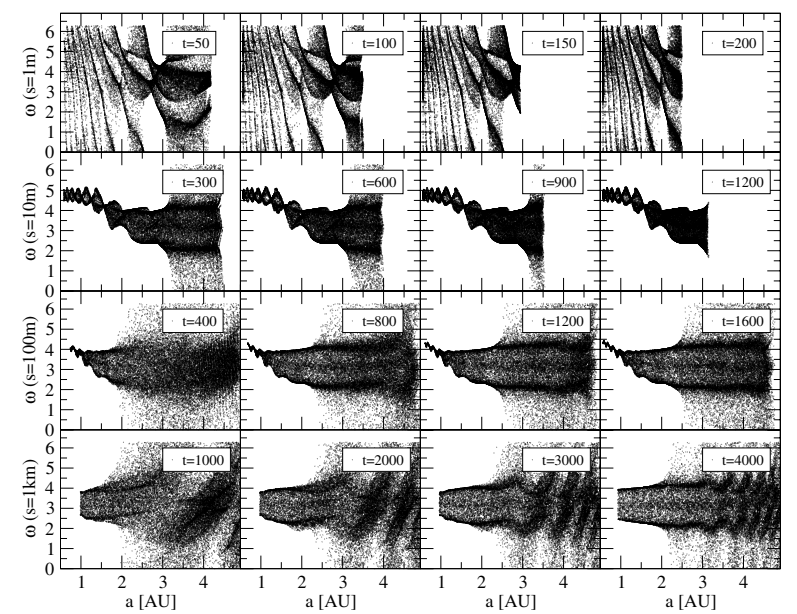

Fig. 8. Periastron longitude $\tilde{\omega}$ in model W2. Each row shows temporal evolution for a particle of a given size. The time is displayed in years.

Figure 8 shows the longitudes of particle periastra with respect to the longitude of the companion, $\tilde{\omega}$, as a function of particle semimajor axis. Each row shows the evolutionary sequence for the population of particles of different size (indicated on the vertical axis legend). The time evolution is shown to prove that the observed configurations are not transient but converge to a certain, stationary pattern on the $\omega-a$ plane.

A quick look at the plot reveals that the effect of periastra alignment is present for all considered sizes of particles. The degree of alignment depends, however, on the distance from the central star and on the particle size. A closer exploration of the plot allows two different regimes to be distinguished depending on the particle size:

1. particles with radii smaller than a few meters feel strong gas drag, and their periastra are correlated with the spiral pattern in the gaseous disk. It can be observed on the plot for $1 \mathrm{~m}$ particles in the region of stable orbits (below $3 \mathrm{AU}$ );

2. particles with radii larger than a few meters have orbits aligned, but the alignment is not correlated with the waves in the gaseous disk. In the outer parts of the disk, around $3 \mathrm{AU}$, the periastra are in opposition to the companion star with a substantial scatter in longitude (larger for larger particles). When the drag force is stronger (in the inner disk or for smaller particles), the alignment is more pronounced. It is particularly strong for $10 \mathrm{~m}$ particles.

We want to stress here that the alignment of periastra does not imply any spatial alignment of particles. In fact we did not observe a spatial correlation of particles with the spiral waves at the particle-size range considered here. We only checked that such a correlation becomes weakly visible for $10 \mathrm{~cm}$ particles.

Figure 9 illustrates the dependence of eccentricity on semimajor axis in the same manner as for periastra longitudes. The coherence in eccentricity is much weaker in comparison to the eccentric model of Marzari \& Scholl (2000). In the inner disk, it is simply the effect of strong damping by the gas drag. The presence of spiral waves manifests mainly for $1 \mathrm{~m}$ and $10 \mathrm{~m}$ size particles in the form of pulsations in $a$. Actually, two pulsation patters can be noticed for $1 \mathrm{~m}$ particles, each corresponding to a spiral arm. We expect that the relative phase between those two patterns depends on the winding angle of the spirals. Thus the coherence in eccentricity will vary depending on the disk temperature.

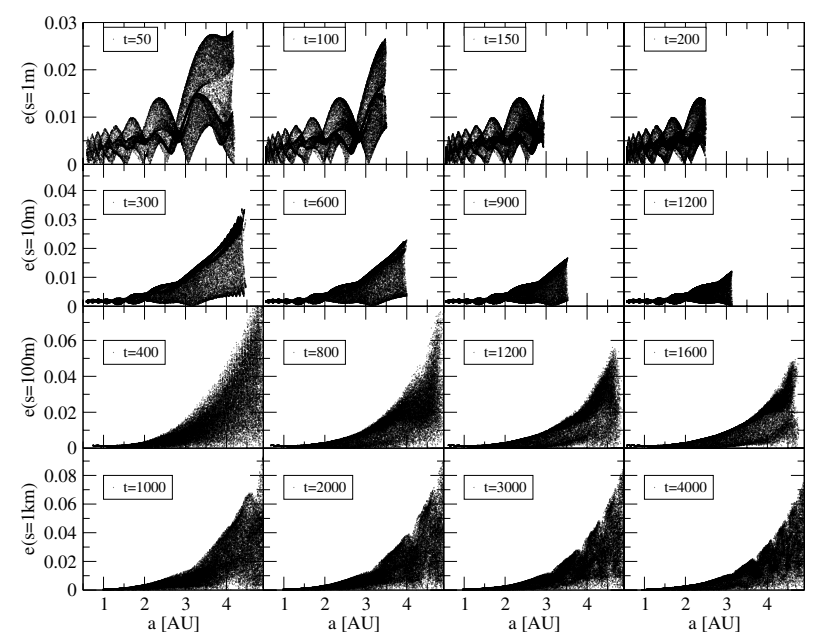

Fig. 9. Eccentricity as a function of semimajor axis. Each row shows the temporal evolution for a particle of a given size.

\section{Discussion and summary}

We have investigated orbital evolution of particles moving in a circumprimary gaseous disk in a circular binary system. This is the first investigation of the orbital motions that includes tidally excited density waves in the gas-drag calculation.

We have demonstrated numerically that the radial flow of gas in the disk (inward or outward) effectively increases the drag force due to an enhanced mass flux colliding with the solid particles. We derived approximate analytical formulae for the change rate of orbital elements $(a, e, i)$ in the gaseous disk, which is not in radial equilibrium. The formulae do not assume anything about the source of the radial gas flow. In general there are four constituents of the relative velocity between particle and gas. The first two, eccentricity and inclination, describe the deviation of the particle from the Keplerian gas flow. The remaining two represent the radial and angular deviation of the gas from the Keplerian flow. To account for the non-axisymmetric features in the disk, one has to introduce effective components of the gas velocity. The effective components can be substantially larger than simple angular averages, meaning that the evolution of orbital elements can be faster in the non-axisymmetric disk. Exactly this situation happens in the investigated disk perturbed by the companion star: the tidally induced spiral waves which propagate radially in the form of shocks increase the effective components of the gas velocity. In particular, in the outer disk where the perturbation is strongest, the effective radial component dominates other components of the relative velocity. We have found numerically that the particles of sizes from $1 \mathrm{~m}$ to $10 \mathrm{~km}$ migrate there around three times faster than in an axisymmetric disk in radial equilibrium. This result agrees with the analytical prediction very well, within a few percent.

The effect of enhanced drag force due to radial gas flow has some significant consequences in the context of planetesimal formation in the binary system. The faster particle migration raises the old question of whether there is enough time to form planetesimals before all smaller particles have fallen onto the star. We have to note, however, that the enhancement in the drag force gradually vanishes inward in the disk. Depending on the disk temperature and density radial profile, this differential migration may eventually result in the accumulation of particles at a certain radius in the inner disk, although we estimate that this is not the case for realistic disk models. There are other effects that may support faster planetesimal formation. If only the 
larger bodies can form quickly, the size-independent enhancement of the migration speed will result in a higher flux of smaller particles, which will feed the larger body. Furthermore, an accelerated damping of the particle's inclinations leads to an increase in their number density in the mid-plane of the disk and more frequent collisions. Both effects are important for the planetesimal formation and early stages of their growth.

The frequency of collisions between particles is only one of the two factors controlling their growth rate. The other one is the relative velocity of colliding bodies that determines whether the bodies stick together or shatter into smaller pieces. The relative velocity almost exclusively depends on the eccentricity and periastra orientation of the particles, provided that the inclinations are already damped. The contribution from decay of the semimajor axis is negligible (in our system it can play a role only for $1 \mathrm{~m}$ particles at $3 \mathrm{AU})$. We find that the spiral waves induce a certain coherency in both periastra longitudes and eccentricities. Only for the smallest particles of $1 \mathrm{~m}$ size does the coherence in periastra longitudes come from a simple correlation of the orbit orientation with the spiral wave pattern in the gas. For larger bodies, there is no such correlation, and the degree of coherence decreases with increasing size of the body. It seems that the relative velocities will be affected by coherence only for bodies smaller than $10 \mathrm{~m}$. In order to determine how much it does help in planetesimal formation, collisional simulations with realistic size distribution will have to be carried. This will be the subject of the next paper.

In this paper we focused on the circular binary system because it allows the sole effects of the spiral waves to be studied without the time dependent effects that are present in the eccentric binary. On the other hand, the radial gas flow in the eccentric system is much stronger and we expect it to influence particle motion to a much higher degree than in the circular case. The eccentric binary case will be investigated in one of the subsequent papers.

Acknowledgements. P.C. acknowledges financial support provided through the European Community's Human Potential Programme under contract HPRN-CT-2002-00308, PLANETS. P.C. and A.G. were supported by the Polish Ministry of Science through grant No. 1 P03D 026 26. The software used in this work was in part developed by the DOE-supported ASC / Alliance Center for Astrophysical Thermonuclear Flashes at the University of Chicago.

\section{Appendix A: Mean variations of orbital elements}

In this section we extend calculations of A76 for the case of a disk in radial non-equilibrium. All other assumptions made in A76 are preserved; in particular, the disk is axisymmetric. Parts of the formulae written in bold font are additions with respect to their formulae.

Introducing non-dimensional radial velocity $\kappa=-V_{r} / v_{\mathrm{k}}$, Eqs. (4.7) in A76 take the following form:

$$
\begin{aligned}
\frac{\mathrm{d} a}{\mathrm{~d} t}= & -A \rho u \frac{2 a}{1-e^{2}}\left\{1+2 e \cos \psi+e^{2}-(1+e \cos \psi)^{3 / 2} h \cos i\right. \\
& \left.+\boldsymbol{\kappa}\left(\mathbf{1}-\boldsymbol{e}^{\mathbf{2}}\right)^{\mathbf{1} / \mathbf{2}} \boldsymbol{e} \sin \psi\right\} \\
\frac{\mathrm{d} e}{\mathrm{~d} t}= & -A \rho u\left\{2 \cos \psi+2 e-\frac{2 \cos \psi+e+e \cos ^{2} \psi}{(1+e \cos \psi)^{1 / 2}} h \cos i\right. \\
& \left.+\boldsymbol{\kappa}\left(\mathbf{1}-\boldsymbol{e}^{\mathbf{2}}\right)^{\mathbf{1} / \mathbf{2}} \sin \psi\right\} \\
\frac{\mathrm{d} i}{\mathrm{~d} t}= & -A \rho u \frac{h}{(1+e \cos \psi)^{1 / 2}} \cos ^{2}(\psi+\omega) \sin i
\end{aligned}
$$

where $\psi$ and $\omega$ denote true anomaly and argument of periastron respectively, and $h \approx 1-\eta$. The total relative velocity $u$ reads:

$$
\begin{aligned}
u^{2}= & v_{\mathrm{k}}^{2}(a)\left\{\left(1-\frac{3}{4} \cos ^{2} \psi\right) e^{2}+\cos ^{2}(\psi+\omega) i^{2}+\eta^{2}+\eta e \cos \psi\right\} \\
& +\frac{\boldsymbol{v}_{\mathbf{k}}^{2}}{\mathbf{1}-\boldsymbol{e}^{\mathbf{2}}}\left\{\boldsymbol{\kappa}^{\mathbf{2}}\left(\mathbf{1}-\boldsymbol{e}^{\mathbf{2}}\right)-\mathbf{2} \boldsymbol{\kappa} \boldsymbol{e}\left(\mathbf{1}-\boldsymbol{e}^{\mathbf{2}}\right)^{\mathbf{1} / \mathbf{2}} \sin \psi\right\} .
\end{aligned}
$$

Assuming that the drag force is weak and the orbital elements are constant during one orbital period, their orbitally averaged rate of variation is expressed as

$\left\langle\frac{\mathrm{d} Q}{\mathrm{~d} t}\right\rangle=\frac{1}{2 \pi} \int_{0}^{2 \pi} \frac{\mathrm{d} Q}{\mathrm{~d} t} \frac{\left(1-e^{2}\right)^{3 / 2}}{(1+e \cos \psi)^{2}} \mathrm{~d} \psi$,

where $Q \in\{a, e, i\}$. In order to simplify further calculations we apply the approximation (Eq. (4.19) in A76):

$\langle u F\rangle=\langle u\rangle\langle F\rangle=\left\{\left\langle u^{2}\right\rangle\right\}^{1 / 2}\langle F\rangle$

where $F$ denotes factors other than $u$ in Eqs. (A.1)-(A.3). It is justified as long as $u$ and $F$ are independent and the constant part of $u^{2}$ is greater than the amplitude of the oscillatory part. Providing that variables $e, i, \eta, \kappa$ are much smaller than unity and preserving only leading terms for each of them, we obtain from Eq. (A.4)

$\left\langle u^{2}\right\rangle=v_{\mathrm{k}}^{2}(a)\left[\left(\frac{5}{8}-\kappa^{2}\right) e^{2}+\frac{1}{2} i^{2}+\eta^{2}+\kappa^{2}\right]$.

In practice, term $\kappa^{2}$ in front of $e^{2}$ is negligible and the remaining formula is intuitive: in addition to the angular component of velocity, $\eta$, its radial part, $\kappa$, enters.

Fortunately, the orbital average of $F$ is not changed with respect to A76, since all new terms in Eqs. (A.1)-(A.3) vanish when averaged. Thus the only changes come from $\left\langle u^{2}\right\rangle$ term and finally we obtain:

$$
\begin{aligned}
& \frac{\tau_{0}}{a}\left\langle\frac{\mathrm{d} a}{\mathrm{~d} t}\right\rangle=-2\left[\left(\frac{5}{8}-\kappa^{2}\right) e^{2}+\frac{1}{2} i^{2}+\eta^{2}+\kappa^{2}\right]^{1 / 2} \eta \\
& \frac{\tau_{0}}{e}\left\langle\frac{\mathrm{d} e}{\mathrm{~d} t}\right\rangle=2 \frac{\tau_{0}}{i}\left\langle\frac{\mathrm{d} i}{\mathrm{~d} t}\right\rangle=\left[\left(\frac{5}{8}-\kappa^{2}\right) e^{2}+\frac{1}{2} i^{2}+\eta^{2}+\kappa^{2}\right]^{1 / 2}
\end{aligned}
$$

\section{References}

Adachi, I., Hayashi, C., \& Nakagawa, K. 1976, Prog. Theor. Phys., 56, 1756 Aarseth, S. J., Turner, E. L., \& Gott, J. R. 1979, ApJ, 228, 664 Agnor, C., \& Asphaug, E. 2004, ApJ, 613, L157

Blondin, J. 2000, New A, 5, 53

Colella, P., \& Woodward, P. R. 1984, JCoPh, 54, 174

Duquennoy, A., \& Mayor, M. 1991, A\&A, 248, 485

Eggenberger, A., Udry, S., \& Mayor, M. 2004, A\&A, 417, 353

Fryxell, B., Olson, K., Ricker, P., et al. 2000, ApJS, 131, 273

Goldreich, P., \& Tremaine, S. 1979, ApJ, 233, 857

Hayashi, C. 1980, Prog. Theor. Phys. Suppl., 70, 35

Heppenheimer, T. A. 1980, A\&A, 65, 421

Inaba, S., Tanaka, H., Nakazawa, K., et al. 2001, Icarus, 149, 235

Kokubo, E., \& Ida, S. 1996, Icarus, 123, 180

Lin, D., \& Papaloizou, J. 1979, MNRAS, 186, 799

Makino, J. 1991, ApJ, 369, 200

Marzari, F., \& Scholl, H. 2000, ApJ, 543, 328

Muterspaugh, M. W., Lane, B. F., Konacki, M., et al. 2006, A\&A, 446, 723

Papaloizou, J., \& Pringle, J. E. 1977, MNRAS, 181, 441

Quintana, E. V., Lissauer, J. J., Chambers, J. E., \& Duncan, M. J. 2002, ApJ, 576,982

Różyczka, M., \& Spruit, H. C. 1993, ApJ, 417, 677

Spruit, H. C. 1987, A\&A, 184, 173

Thébault, P., Marzari, F., Scholl, H., et al. 2004, A\&A, 427, 1097

Thébault, P., Marzari, F., \& Scholl, H. 2006, Icarus, 183, 193

Whitmire, D., Matese, J., \& Criswell, L. 1998, Icarus, 132, 196

Zucker, S., \& Mazeh, T. 2002, ApJ, 568, L113 